\title{
The usefulness of the Rotarex thrombectomy system in the recanalization of an occluded covered stent in patients after cavo-pulmonary (Glenn) shunt
}

\author{
Paweł J. Cześniewicz ${ }^{1}$, Jacek Kusa ${ }^{1,2}$ \\ ${ }^{1}$ Pediatric Cardiology Department, Regional Specialist Hospital, Research and Development Centre, Wroclaw, Poland \\ ${ }^{2}$ Pediatric Cardiology Department, Medical University of Silesia, Katowice, Poland
}

Adv Interv Cardiol 2019; 15, 3 (57): 377-379

DOI: https://doi.org/10.5114/aic.2019.87900

The present report describes a novel approach to instent thrombosis in the left pulmonary artery treated with the Rotarex thrombectomy system in patients with single ventricle anatomy after cavo-pulmonary (Glenn) shunt.

We report a case of a 13-year-old girl with single ventricle anatomy (a double outlet right ventricle with a hypoplastic left ventricle) after cavo-pulmonary (Glenn) shunt, who was referred to our hospital with deteriorating exercise capacity and low arterial oxygen saturation (75\%).

Cardiac catheterization was performed and angiography demonstrated left pulmonary artery stenosis with significant antegrade pulmonary flow from a single ventricle (Figure 1 A). A 12 Fr Mullins long sheath was introduced and a covered stent (CP 8ZIG $45 \mathrm{~mm}$, NuMED) mounted on a $14 \mathrm{~mm}$ diameter BiB balloon (NuMED) was implanted in the middle portion of the left pulmonary artery, and thus the antegrade pulmonary flow was closed and the pulmonary stenosis was resolved (Figure 1 B). Standard anti-platelet therapy with acetylsalicylic acid was introduced and the patient was discharged 6 days later. The patient was considered as a candidate for a Fontan operation and the next cardiac catheterization was planned in 6 months.

Three months later the patient was admitted to our hospital again with signs of progressive cyanosis (arterial oxygen saturation: $65 \%$ ).

Urgent cardiac catheterization was performed and angiography demonstrated an occlusion of the previously implanted stent in the left pulmonary artery (Figure $1 \mathrm{C}$ ). Conservative medical management with heparin was administered. After 1 week of therapy, no clinical evidence of improvement had occurred. Because the patient was considered a poor surgical candidate due to numerous previous sternotomies, the decision to recan- alize the left pulmonary artery with the Rotarex (Straub Medical) thrombectomy system was made. The occluded vessel was crossed through with a 0.014" Asato guidewire (Asahi Intecc) and a $4 \mathrm{Fr}$ vertebral catheter. Multiple passes through the thrombus were made with different catheters and guidewires; thus partial mechanical fragmentation of the thrombus was performed. An $8 \mathrm{Fr}$ Rotarex catheter was advanced and multiple blood clots were aspirated and removed. Subsequently, an 18-mm diameter Atlas Gold balloon (Bard Medical) was inserted and inflated. Control angiography showed complete recanalization of the left pulmonary artery, but inside its branches thrombi were still present. For this reason, numerous aspirations of this material were made, resulting in a large amount of embolic material. In subsequent angiography, the flow to all segments of the left lung was confirmed (Figure $1 \mathrm{D}$ ). After the procedure, a blood transfusion was required. Standard anticoagulation and antiplatelet treatments were initiated. In the following days, we observed slightly higher arterial oxygen blood saturation of $75-80 \%$ and better exercise capacity.

Pulmonary artery stenosis associated with a significant anterograde pulmonary flow is rarely found in patients with single ventricle anatomy after the Glenn operation. Covered stent implantation resolves both problems and this procedure has been recently described [1]. Nevertheless, a nonpulsative, slow pulmonary blood flow, a high hematocrit level and PTFE covering of the stent create favorable circumstances for in-stent thrombosis.

A conservative therapeutic strategy for in-stent thrombosis includes anticoagulant or thrombolytic therapy. If pharmacological reperfusion therapy has failed, surgical embolectomy should be deliberated. Although pulmonary embolectomy is considered a technically sim- 

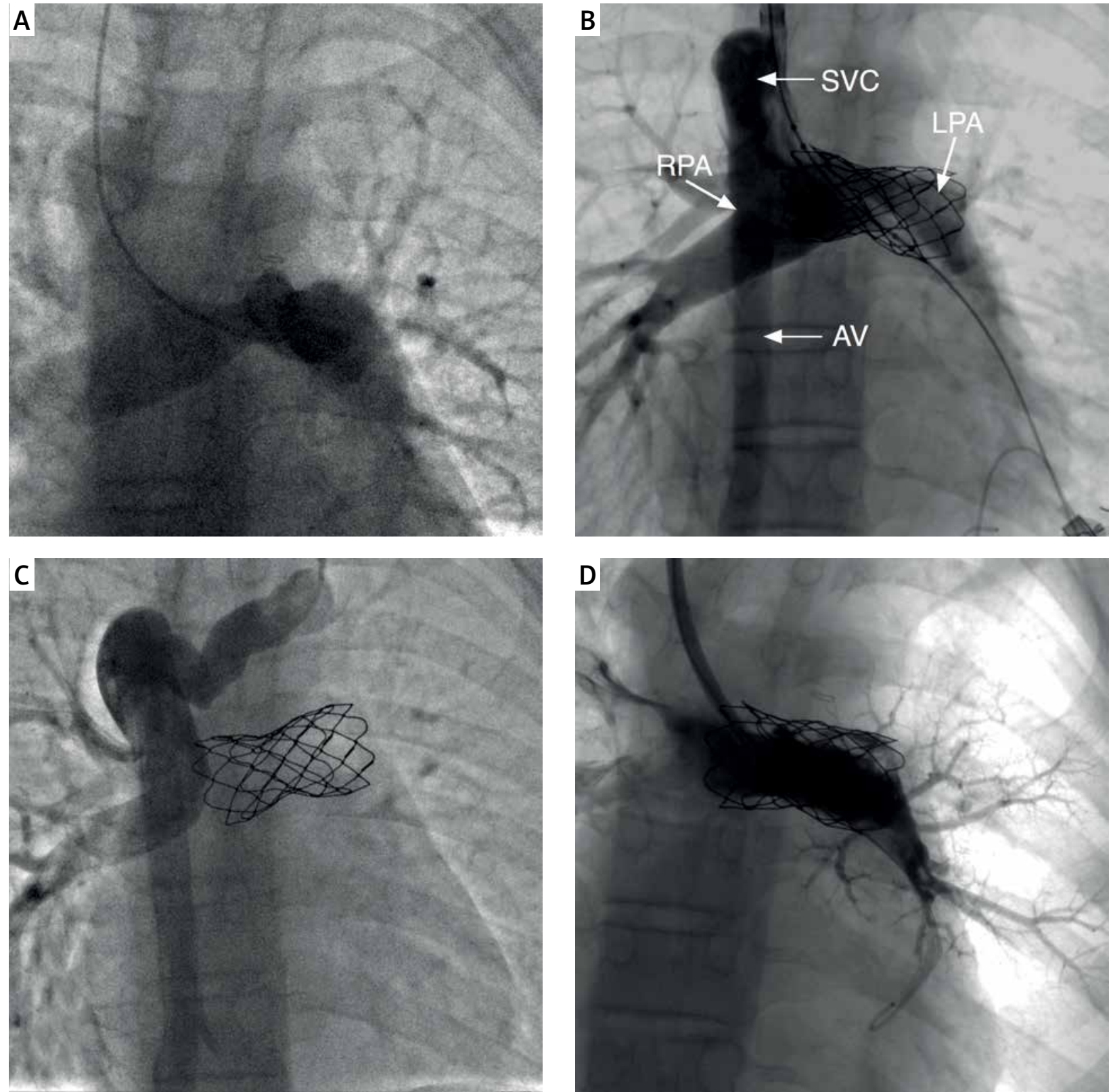

Figure 1. A - The pulmonary angiography shows left pulmonary artery stenosis. B - The covered stent (CP 8ZIG $45 \mathrm{~mm}$, NuMED) was implanted in the pulmonary artery. Reverse flow is visible in the azygos vein. $\mathbf{C}-$ The angiography performed 3 months later reveals the occlusion of a previously implanted stent. D - The final angiography reveals almost complete resolution of the in-stent thrombus and restoration of the circulation to segmental arteries of the left pulmonary artery

SVC - superior vena cava, AV - azygos vein, RPA - right pulmonary artery, LPA - left pulmonary artery.

ple operation, patients with numerous previous sternotomies appear to have a worse outcome. Alternatively, catheter-based mechanical thrombectomy is one of the treatment options. However, it has been rarely reported in patients with single ventricle anatomy $[2,3]$.

Hirono reported two cases of percutaneous mechanical thrombectomy for in-stent thrombosis in the pulmonary artery in patients after Fontan surgery [2]. In both cases, the mechanical thrombectomy system was used for the treatment of a recent thrombus, which formed between ten days and one month after stent implantation. Only in the first case did the pulmonary artery remain patent. The time from the beginning of clot formation to intervention may be crucial. Even 2 weeks after the incident, the thrombus may be partly converted to fibrous connective tissue. In these circumstances, anticoagulation and thrombolysis therapy are ineffective. Also, mechanical thrombectomy shows the best effectiveness 
in a newly formed thrombus; nevertheless, we achieved a satisfactory effect in patients 3 months after stent implantation.

One should pay attention to the fact that the Rotarex thrombectomy system is generally intended for use in peripheral arteries due to the extraordinary suction power of this device [4]. In our opinion, this device is both safe and effective in pulmonary arteries when treating occlusion in a previously implanted stent.

In conclusion, mechanical thrombectomy for in-stent thrombosis in children constitutes an alternative option when conservative treatment is ineffective and surgery is contraindicated.

\section{Conflict of interest}

The authors declare no conflict of interest.

\section{References}

1. Butera G, Taha F. Two problems and a single solution: covered stent implantation to close an anterograde pulmonary flow and treat hypoplastic left pulmonary artery after Fontan operation. Catheter Cardiovasc Interv 2016; 87: E240-2.

2. Menon SC, Hagler DJ, Cetta F, et al. Rheolytic mechanical thrombectomy for pulmonary artery thrombus in children with complex cyanotic congenital heart disease. Catheter Cardiovasc Interv 2008; 71: 237-43.

3. Hirono K, Ibuki K, Tomita H. Percutaneous catheter aspiration thrombectomy for the occluded stents of pulmonary artery in children with single ventricle physiology after fontan surgery. Catheter Cardiovasc Interv 2014; 84: 1153-6.

4. Stanek F, Ouhrabkova R, Prochazka D. Mechanical thrombectomy using the Rotarex catheter - safe and effective method in the treatment of peripheral arterial thromboembolic occlusions. Vasa 2010; 39: 334-40. 